\title{
A RESTRICTED CLASS OF CONVEX FUNCTIONS
}

CECIL L. WOODS

1. Introduction. The relationship of the inequalities

$$
I(f, x, h, \alpha) \leqq A(f, x, h, \beta)
$$

and

$$
I(f, x, h, \alpha) \geqq A(f, x, h, \beta)
$$

to general convex and concave functions respectively has been treated by Radó [1]. ${ }^{1}$ In his and in this paper, $f(x)$ denotes a positive continuous function defined on a given open interval $x_{1}<x<x_{2}$. In $I(f, x, h, \alpha)$ and $A(f, x, h, \beta)$, defined by Radó $[1, \mathrm{pp} .267,268]$ as follows:

$$
\begin{aligned}
& I(f, x, h, \alpha)=\left[\frac{1}{2 h} \int_{-h}^{h} f(x+\xi)^{\alpha} d \xi\right]^{1 / \alpha}, \quad \text { if } \alpha \neq 0, \\
& I(f, x, h, 0)=\exp \left[\frac{1}{2 h} \int_{-h}^{h} \log f(x+\xi) d \xi\right], \\
& A(f, x, h, \beta)=\left[\frac{f(x-h)^{\beta}+f(x+h)^{\beta}}{2}\right]^{1 / \beta}, \quad \text { if } \beta \neq 0, \\
& A(f, x, h, 0)=\exp \left[\frac{\log f(x-h)+\log f(x+h)}{2}\right] \\
& =[f(x-h) f(x+h)]^{1 / 2},
\end{aligned}
$$

$x$ and $h$ satisfy the inequalities $x_{1}<x-h<x+h<x_{2} ; \alpha$ and $\beta$ are real exponents.

To enable us to express his definitions and results concisely, we define four classes of $f(x)$ as follows:

Let $K$ be the class of all $f(x)$ which are convex on $x_{1}<x<x_{2}$.

Let $K_{\alpha \beta}$ be the class of all $f(x)$ which satisfy the inequality (1.1).

Let $K^{*}$ be the class of all $f(x)$ which are concave on $x_{1}<x<x_{2}$.

Let $K_{\alpha \beta}^{*}$ be the class of all $f(x)$ which satisfy the inequality (1.2). Then the four sets of pairs $(\alpha, \beta)$ which Rado defined [1, pp. 269, 281] can be defined by the statements:

Let $E$ be the set of all pairs $(\alpha, \beta)$ for which $K \subset K_{\alpha \beta}$.

Let $E$ be the set of all pairs $(\alpha, \beta)$ for which $K_{\alpha \beta} \subset K$.

Let $E^{*}$ be the set of all pairs $(\alpha, \beta)$ for which $K^{*} \subset K_{\alpha \beta}^{*}$.

Received by the editors August 24, 1945.

1 Numbers in brackets indicate the references at the end of the paper. 
Let $\bar{E}^{*}$ be the set of all pairs $(\alpha, \beta)$ for which $K_{\alpha \beta}^{*} \subset K^{*}$.

We also use two of the functions of $\alpha$ defined by Radó [1, p. 269, footnote]:

$$
\begin{aligned}
& \psi_{2}=\psi_{2}(\alpha)=(\alpha+2) / 3, \\
& \psi_{3}=\psi_{3}(\alpha)=\left\{\begin{array}{lr}
0, & -\infty<\alpha<+\infty, \\
\frac{\alpha \log 2}{\log (\alpha+1)}, & -1<\alpha<\infty, \alpha \neq 0, \\
\log 2, & \alpha=0,
\end{array}\right.
\end{aligned}
$$

to give his theorems [1, pp. 269, 281, 282]:

THEOREM 1.61. A pair $(\alpha, \beta)$ belongs to $E$ if and only if $\beta \geqq \max \left(\psi_{2}, \psi_{3}\right)$.

TheOrem 1.62. $A$ pair $(\alpha, \beta)$ belongs to $\bar{E}$ if and only if $\beta \leqq \psi_{2}$.

THEOREM 1.63. A pair $(\alpha, \beta)$ belongs to $E^{*}$ if and only if $\beta \leqq \min \left(\psi_{2}, \psi_{3}\right)$.

Theorem 1.64. $A$ pair $(\alpha, \beta)$ belongs to $\bar{E}^{*}$ if and only if $\beta \geqq \psi_{2}$.

From Theorems 1.61 and 1.63 it is easily seen that $E$ and $E^{*}$ do not cover the $\alpha \beta$-plane. This leads us to an investigation of the inequalities (1.1) and (1.2) for pairs $(\alpha, \beta)$ not included in $E$ or $E^{*}$ and thus to a restriction on the classes $K$ and $K^{*}$. For each $M>0$, we define a class $K_{M}$ as follows:

Let $K_{M}$ be the class of all $f(x)$ such that $2^{-1} \log (B / b) \leqq M$, where $B$ and $b$ are respectively the least upper bound and greatest lower bound of $f(x)$ on $x_{1}<x<x_{2}$.

Then we define two sets of pairs $(\alpha, \beta)$ by the statements:

Let $E_{M}$ be the set of all pairs $(\alpha, \beta)$ for which $K K_{M} \subset K_{\alpha \beta}$.

Let $E_{M}{ }^{*}$ be the set of all pairs $(\alpha, \beta)$ for which $K^{*} K_{M} \subset K_{\alpha \beta}^{*}$.

As we shall show, sets $E_{M}$ and $E_{M}{ }^{*}$ increase as $M$ decreases, and $E$ and $E^{*}$ are proper subsets of $E_{M}$ and $E_{M}{ }^{*}$ respectively.

2. A word as to method. The inequality (1.1) can obviously be written

$$
\log \frac{I(f, x, h, \alpha)}{A(f, x, h, \beta)} \leqq 0 .
$$

We follow Radó in investigating first the special case where $f(x)$ is a linear function $l(x) . I(l, x, h, \alpha)$ can be computed for all $\alpha$, and we choose a variable $v$ defined by 


$$
v=\frac{1}{2} \log \frac{l(x+h)}{l(x-h)},
$$

by which choice the left member of (2.1) in this case is transformed into a function $\phi(v, \alpha, \beta)$ defined for all $v, \alpha$, and $\beta$. We shall explicitly compute $\phi(v, \alpha, \beta)$ and investigate a number of its significant properties. On the basis of this investigation we shall separate the $\alpha \beta$-plane into point sets for each of which $\phi(v, \alpha, \beta)$ has certain invariant properties for all $(\alpha, \beta)$ in the set. We shall draw curves $\phi=\phi(v, \alpha, \beta)$ in the $v \phi$-plane which exhibit these invariant properties. Finally we shall state and prove two theorems about $E_{M}$ and $E_{M}{ }^{*}$.

3. $I, A$, and $\phi$ expressed as functions of $v$. In the case where $f(x)$ is linear, we let $A_{0}$ denote $[l(x-h) l(x+h)]^{1 / 2}$. Performing the integrations of (1.3), factoring out $A_{0}$ from the results and from (1.4), and substituting $e^{2 v}$ for $l(x+h) / l(x-h)$ according to (2.2), we obtain, for $v \neq 0$,

$$
\begin{array}{rlrl}
I(l, x, h, \alpha) & =A_{0}\left[\frac{\sinh (\alpha+1) v}{(\alpha+1) \sinh v}\right]^{1 / \alpha}, & & \text { if } \alpha \neq 0,-1, \\
I(l, x, h,-1) & =A_{0}(\sinh v) / v, & \\
I(l, x, h, 0) & =A_{0} \exp (v \operatorname{ctnh} v-1), & & \\
A(l, x, h, \beta) & =A_{0}(\cosh \beta v)^{1 / \beta}, & & \text { if } \beta \neq 0, \\
A(l, x, h, 0) & =A_{0} . &
\end{array}
$$

Since $\phi(v, \alpha, \beta)$ is the logarithm of the ratio of $I(l, x, h, \alpha)$ to $A(l, x, h, \beta)$, we see that $\phi(v, \alpha, \beta)$ is independent of $A_{0}$. From (3.1) and (3.2) we have, for $v \neq 0$,

$$
\phi(v, \alpha, \beta)=\frac{1}{\alpha} \log \frac{\sinh (\alpha+1) v}{(\alpha+1) \sinh v}-\frac{1}{\beta} \log \cosh \beta v,
$$

$$
\text { if } \alpha \neq 0,-1 ; \beta \neq 0 \text {, }
$$

$$
\begin{array}{rlrl}
\phi(v, 0, \beta) & =v \operatorname{ctnh} v-1-(1 / \beta) \log \cosh \beta v, & & \text { if } \beta \neq 0, \\
\phi(v,-1, \beta) & =\log ((\sinh v) / v)-(1 / \beta) \log \cosh \beta v, & \text { if } \beta \neq 0, \\
\phi(v, \alpha, 0) & =\frac{1}{\alpha} \log \frac{\sinh (\alpha+1) v}{(\alpha+1) \sinh v}, & \text { if } \alpha \neq 0,-1, \\
\phi(v, 0,0) & =v \operatorname{ctnh} v-1, & \\
\phi(v,-1,0) & =\log ((\sinh v) / v) ; &
\end{array}
$$


and

$$
\phi(0, \alpha, \beta)=0 \text {. }
$$

That $\phi(0, \alpha, \beta)=0$ follows trivially from (2.2), for $v=0$ implies that $l(x)$ is constant, and each of the means in (1.3) and (1.4) has the same constant value. We observe that $\phi(v, \alpha, \beta)$ is a continuous function of $v, \alpha$, and $\beta$ for all finite values of the independent variables. We also see that $\phi(v, \alpha, \beta)$ is an even function of $v$. Hence we shall in what follows consider $v>0$.

4. The set in which $\phi(v, \alpha, \beta)$ is identically zero. We have the following result.

THEOREM 4.1. In the set of just three points which form the complete intersection of $E$ and $E^{*}$, namely: $(-2,0),(-1 / 2,1 / 2)$, and $(1,1)$, $\phi(v, \alpha, \beta) \equiv 0$.

The proof for the point $(-2,0)$ lies in the fact that $\sinh v$ is an odd function, and for the other two points in the identity: $\sinh 2 v$ $=2 \sinh v \cosh v$. That there are no other points where $\phi(v, \alpha, \beta) \equiv 0$ follows from the facts developed in the next section.

5. The character of $\phi(v, \alpha, \beta)$ for sufficiently small $v$. For sufficiently small positive $v$, and, because of the continuity of $\phi(v, \alpha, \beta)$, for all $\alpha$ and $\beta$, we have the following expansion of $\phi(v, \alpha, \beta)$ in power series of $v$ :

$$
\begin{aligned}
\phi(v, \alpha, \beta)= & (\alpha+2-3 \beta) v^{2} / 6 \\
& -\left(\alpha^{3}+4 \alpha^{2}+6 \alpha+4-15 \beta^{3}\right) v^{4} / 180+O\left(v^{0}\right) .
\end{aligned}
$$

This is easily verified by integrating the series for ctnh $v$ and $\tanh v$, and applying the results to (3.3).

From (5.1) we obtain the following theorem:

THEOREM 5.2. There exists an $\epsilon>0$ such that for $0<v<\epsilon$,

(1) $\phi(v, \alpha, \beta)>0$, if $\alpha+2-3 \beta>0$, or if $\alpha+2-3 \beta=0$, and $-\infty<\alpha$ $<-2 ;-1 / 2<\alpha<1$,

(2) $\phi(v, \alpha, \beta)<0$, for all other pairs $(\alpha, \beta)$ except those in $E E^{*}$.

The proof consists in the following remarks: for sufficiently small $v$,

(1) $\operatorname{sgn} \phi(v, \alpha, \beta)=\operatorname{sgn}(\alpha+2-3 \beta)$, if $\alpha+2-3 \beta \neq 0$,

(2) $\operatorname{sgn} \phi(v, \alpha,(\alpha+2) / 3)=-\operatorname{sgn}(\alpha-1)(2 \alpha+1)(\alpha+2)$, if $\alpha \neq-2$, $-1 / 2$, or 1 .

6. The character of $\phi(v, \alpha, \beta)$ as $v$ approaches infinity. We shall show that $\lim _{v \rightarrow \infty} \phi(v, \alpha, \beta)$ is not a continuous function of $\alpha$ and $\beta$ for all values of $\alpha$ and $\beta$. 
From definitions we obtain the following identities:

$$
\begin{aligned}
\log \sinh v & =v+\log \left(1-e^{-2 v}\right)-\log 2, \\
\log \cosh v & =v+\log \left(1+e^{-2 v}\right)-\log 2, \\
\operatorname{ctnh} v & =1+2 /\left(e^{2 v}-1\right) .
\end{aligned}
$$

We use (6.1), (6.2), and (6.3) to express $\phi(v, \alpha, \beta)$ for large positive $v$. We write only those terms which are significant as $v \rightarrow \infty$, and indicate by three dots the omission of two kinds of terms: (1) terms involving exponentials which vanish as $\nu \rightarrow \infty$, and (2) constants and terms in $\alpha$ and $\beta$, independent of $v$, in those cases where $\lim _{v \rightarrow \infty} \phi(v, \alpha, \beta)$ is positive infinity or negative infinity.

We have, for $\beta>0$,

$$
\begin{aligned}
\phi(v, \alpha, \beta)=(1 / \beta) \log 2-(1 / \alpha) \log (\alpha+1) & +\cdots, \\
& \text { if } \alpha>-1, \alpha \neq 0,
\end{aligned}
$$

(6.4) $\phi(v, 0, \beta)=(1 / \beta) \log 2-1+\cdots$,

$$
\phi(v,-1, \beta)=-\log v+\cdots,
$$

$$
\phi(v, \alpha, \beta)=-2(\alpha+1) v / \alpha+\cdots, \quad \text { if } \alpha<-1 \text {; }
$$

for $\beta=0$,

$$
\begin{array}{rlrl}
\phi(v, \alpha, 0) & =v+\cdots, & & \text { if } \alpha>-1, \alpha \neq 0, \\
\phi(v, 0,0) & =v+\cdots, & \\
\phi(v,-1,0) & =v-\log v+\cdots, & & \text { if } \alpha<-1 ; \\
\phi(v, \alpha, 0) & =-(\alpha+2) v / \alpha+\cdots, &
\end{array}
$$

and for $\beta<0$,

$$
\begin{array}{rlrl}
\phi(v, \alpha, \beta) & =2 v+\cdots, & & \text { if } \alpha>-1, \alpha \neq 0, \\
\phi(v, 0, \beta) & =2 v+\cdots, & \\
\phi(v,-1, \beta) & =2 v-\log v+\cdots, & & \\
\phi(v, \alpha, \beta) & =-2 v / \alpha+\cdots, & & \text { if } \alpha<-1 .
\end{array}
$$

From (6.4), (6.5), and (6.6) we conclude:

THEOREM 6.7. As v approaches infinity:

(1) $\lim \phi(v, \alpha, \beta)=+\infty$, for (1) $\beta<0$ and all $\alpha$; and (2) $\beta=0$, $\alpha>-2$;

(2) $\lim \phi(v, \alpha, \beta)=-\infty$, for (1) $\beta=0, \alpha<-2$; and (2) $\beta>0, \alpha \leqq-1$;

(3) $\lim \phi(v, \alpha, \beta)=(1 / \beta) \log 2-(1 / \alpha) \log (\alpha+1)$, for $\beta>0, \alpha>-1$, $\alpha \neq 0$;

(4) $\lim \phi(v, 0, \beta)=(1 / \beta) \log 2-1$, for $\beta>0$. 
We observe that for $\alpha>-1, \beta>0, \lim _{v \rightarrow \infty} \phi(v, \alpha, \beta)$ is a continuous function of $\alpha$ and $\beta$, and the limit is positive, zero, or negative according as $\beta$ is respectively less than, equal to, or greater than $\psi_{3}(\alpha)$, $\alpha>-1$.

7. The number of extrema of $\phi(v, \alpha, \beta)$ for positive $v$. We shall define

$$
\psi_{4}=\psi_{4}(\alpha)=\left\{\begin{array}{rc}
0, & -\infty<\alpha \leqq-1, \\
\alpha+1, & -1<\alpha \leqq 0, \\
1, & 0<\alpha<+\infty,
\end{array}\right.
$$

and then state and prove the following theorem.

THEOREM 7.2. For $v>0$, and $(\alpha, \beta) \neq(-2,0),(-1 / 2,1 / 2)$ or $(1,1)$, there is at most one extremum of $\phi(v, \alpha, \beta)$, and an extremum exists if and only if the pair $(\alpha, \beta)$ satisfies the inequality: $\min \left(\psi_{2}, \psi_{4}\right)<\beta$ $<\max \left(\psi_{2}, \psi_{4}\right)$.

Except for the three $(\alpha, \beta)$-points where $\phi(v, \alpha, \beta)$ vanishes identically, $\phi_{v}(v, \alpha, \beta)$ has at most one zero for positive $v$. We shall show this, and determine the point set in which a zero exists. Differentiating $\phi(v, \alpha, \beta)$ in (3.3), we have, for $v \neq 0$,

$$
\begin{array}{rlr}
\phi_{v}(v, \alpha, \beta)= & (1 / \alpha)[(\alpha+1) \operatorname{ctnh}(\alpha+1) v-\operatorname{ctnh} v] \\
& -\tanh \beta v, \quad \alpha \neq 0,-1 ; \beta \neq 0, \\
\phi_{v}(v, 0, \beta)= & \operatorname{ctnh} v-v \operatorname{csch}^{2} v-\tanh \beta v, \quad \beta \neq 0, \\
(7.3) \phi_{v}(v,-1, \beta)= & \operatorname{ctnh} v-1 / v-\tanh \beta v, \quad \beta \neq 0, \\
\phi_{v}(v, \alpha, 0)= & (1 / \alpha)[(\alpha+1) \operatorname{ctnh}(\alpha+1) v-\operatorname{ctnh} v], \\
\phi_{v}(v, 0,0)= & \operatorname{ctnh} v-v \operatorname{csch}^{2} v=\left(2^{-1} \sinh 2 v-v\right) / \sinh ^{2} v, \\
\phi_{v}(v,-1,0)= & \operatorname{ctnh} v-1 / v=(v-\tanh v) /(v \tanh v) .
\end{array}
$$

We observe that $\phi_{v}(v, \alpha, \beta)$ is a continuous function of $\alpha$ and $\beta$, for all finite $v$. It is easily verified that $\lim _{v \rightarrow 0} \phi_{v}(v, \alpha, \beta)=0$, a fact which is more easily seen from (5.1). Expressing $\phi_{v}(v, \alpha, \beta)$ in terms of hyperbolic sines and cosines only, combining the terms into one fraction, and simplifying the numerators by replacing products of hyperbolic functions by equivalent sums [2, p. 129], we obtain

$$
\phi_{v}(v, \alpha, \beta)=\frac{\alpha \sinh (\alpha-\beta+2) v-(\alpha+1) \sinh (\alpha-\beta) v-\sinh (\alpha+\beta) v}{2 \alpha \sinh (\alpha+1) v \sinh v \cosh \beta v}
$$

$$
\alpha \neq 0,-1 ; \beta \neq 0,
$$




$$
\phi_{v}(v, 0, \beta)=\frac{\sinh (2-\beta) v+\sinh \beta v-2 v \cosh \beta v}{2 \sinh ^{2} v \cosh \beta v}, \quad \beta \neq 0,
$$

$$
\begin{aligned}
\phi_{v}(v,-1, \beta) & =\frac{2 v \cosh (\beta-1) v-\sinh (\beta+1) v+\sinh (\beta-1) v}{2 v \sinh v \cosh \beta v}, \beta \neq 0, \\
\phi_{v}(v, \alpha, 0) & =\frac{\alpha \sinh (\alpha+2) v-(\alpha+2) \sinh \alpha v}{2 \alpha \sinh (\alpha+1) v \sinh v}, \quad \alpha \neq 0,-1 .
\end{aligned}
$$

We do not modify $\phi_{v}(v, 0,0)$ and $\phi_{v}(v,-1,0)$ in this way, for we easily see from the final expressions for them in (7.3) that they are never zero for $v>0$.

We remark that $\phi_{v}(v, \alpha, \beta)$ has a zero for positive $v$ if and only if the numerator in the right member of an equation of (7.4) is zero for some positive value of $v$. Letting $N(v, \alpha, \beta)$ denote this numerator, we may write, for $\alpha \neq 0,-1 ; \beta \neq 0$,

$$
\begin{aligned}
2 N(v, \alpha, \beta)= & \alpha e^{(\alpha-\beta+2) v}-\alpha e^{-(\alpha-\beta+2) v}+(\alpha+1) e^{(\beta-\alpha) v} \\
& -(\alpha+1) e^{-(\beta-\alpha) v}+e^{-(\alpha+\beta) v}-e^{(\alpha+\beta) v}
\end{aligned}
$$

We observe that $N(v, \alpha, \beta)$ is identically zero for all $v$ if $(\alpha, \beta)$ is in $E E^{*}$. For all other $(\alpha, \beta)$ pairs, the equation $N(v, \alpha, \beta)=0$ has at most five roots counting each root with its proper multiplicity [3, (77), p. 49]. $N(v, \alpha, \beta)$ is obviously an odd function of $v$, and we shall show that $N(v, \alpha, \beta)=0$ has a triple root for $v=0$, and hence it has at most one positive root. Using the series for hyperbolic sine, we expand $N(v, \alpha, \beta)$ in powers of $v$, the linear terms vanish, and we have

$$
\begin{aligned}
& N(v, \alpha, \beta)=4 \alpha(\alpha+1)(\alpha+2-3 \beta) v^{3} / 3 !+O\left(v^{5}\right) \text {, } \\
& \text { if } \alpha \neq 0,-1 ; \beta \neq 0 ; \alpha+2-3 \beta \neq 0 \text {, } \\
& N(v, \alpha,(\alpha+2) / 3)=-(64 / 27) \alpha(\alpha+1)(\alpha+2)(\alpha+1 / 2)(\alpha-1) v^{5} / 5 \text { ! } \\
& +O\left(v^{7}\right) \text {, } \\
& \text { if } \alpha \neq 0,-1 \text {. }
\end{aligned}
$$

Inspection of (7.6) shows that $N(v, \alpha, \beta)=0$ has a triple root for $v=0$ if $\alpha \neq 0,-1 ; \beta \neq 0$; and $\alpha+2-3 \beta \neq 0$. The multiplicity is five if $\alpha+2-3 \beta=0$. Hence a positive root exists only if $\alpha+2-3 \beta \neq 0$. Now for sufficiently small $v$,

$$
\begin{aligned}
& \operatorname{sgn} N(v, \alpha, \beta)=\operatorname{sgn} \alpha(\alpha+1)(\alpha+2-3 \beta), \\
& \alpha \neq 0,-1 ; \beta \neq 0 ; \alpha+2-3 \beta \neq 0,
\end{aligned}
$$

as one sees from (7.6). As $v$ approaches infinity, $N(v, \alpha, \beta)$ is dominated by the term having the largest exponent in the right member of (7.5). Hence as $v \rightarrow \infty$, 


$$
\operatorname{sgn} N(v, \alpha, \beta)=\operatorname{sgn} \alpha(\alpha+1)\left(\psi_{4}-\beta\right), \quad \alpha \neq 0,-1 .
$$

Plotting the signs of $N(v, \alpha, \beta)$ for small $v$ and for large $v$, in the $\alpha \beta$-plane, we find that they are opposite in and only in those point sets specified in Theorem 7.2. If $\beta=0,1$ or $\alpha+1$, the number of terms of $N(v, \alpha, \beta)$ in (7.5) having different exponents is four, and hence there are at most three roots. Again $v=0$ is a triple root of $N(v, \alpha, \beta)$ $=0$, and there are no positive roots.

A similar investigation for $\alpha=-1$ or $\alpha=0$ shows that a positive root exists for $N(v, \alpha, \beta)=0$, if $0<\beta<1 / 3$ or $2 / 3<\beta<1$ respectively.

8. The number of zeros of $\phi(v, \alpha, \beta)$ for positive $v$. We state the following result.

Theorem 8.1. A zero of $\phi(v, \alpha, \beta)$ exists for positive $v$ if and only if

$$
\min \left(\psi_{2}, \psi_{3}\right) \leqq \beta \leqq \max \left(\psi_{2}, \psi_{3}\right),
$$

where either both equalities hold or neither holds, and the zero is unique if neither equality holds.

The case where both equalities hold follows from Theorem 4.1 , for $\psi_{2}(\alpha)$ intersects $\psi_{3}(\alpha)$ in $(-2,0),(-1 / 2,1 / 2)$, and $(1,1)$. That a zero exists when neither equality holds follows from the continuity of $\phi(v, \alpha, \beta)$ and Theorems 5.2 and 6.7, since $\operatorname{sgn} \phi(v, \alpha, \beta)$ for small $v$ is opposite to that for large $v$ if and only if both inequalities in (8.2) hold. The necessity of the condition (8.2) and the uniqueness of the zero follow from the facts: $\phi(0, \alpha, \beta)=0$, and when an extremum exists it is unique.

9. The character of $\phi(v, \alpha, \beta)$ at all points of the $\alpha \beta$-plane. In fig-

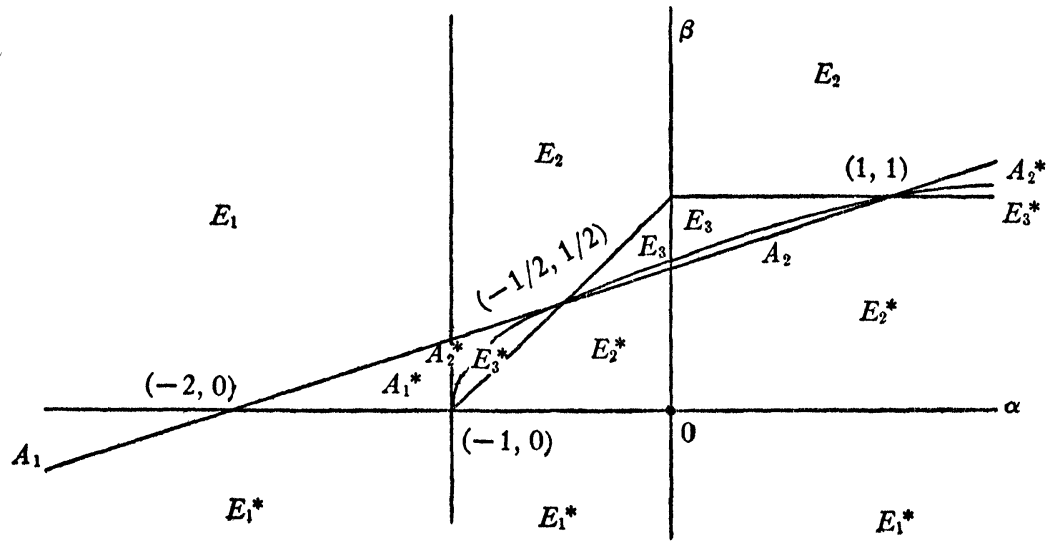

Frg. 1 
ure 1 we have drawn the $\alpha \beta$-plane separated into sets and subsets for each of which $\phi(v, \alpha, \beta)$ has the following properties: (1) for sets:

(1) $\operatorname{sgn} \phi(v, \alpha, \beta)$ for sufficiently small $v$ is invariant,

(2) $\operatorname{sgn} \phi(v, \alpha, \beta)$ for sufficiently large $v$ is invariant,

(3) the number of zeros of $\phi(v, \alpha, \beta)$ is invariant, and in addition to these three, we have (2) for subsets,

(4) $\lim _{v \rightarrow \infty} \phi(v, \alpha, \beta)$ is invariantly positive or negative, finite or infinite,

(5) the number of extrema is invariant.

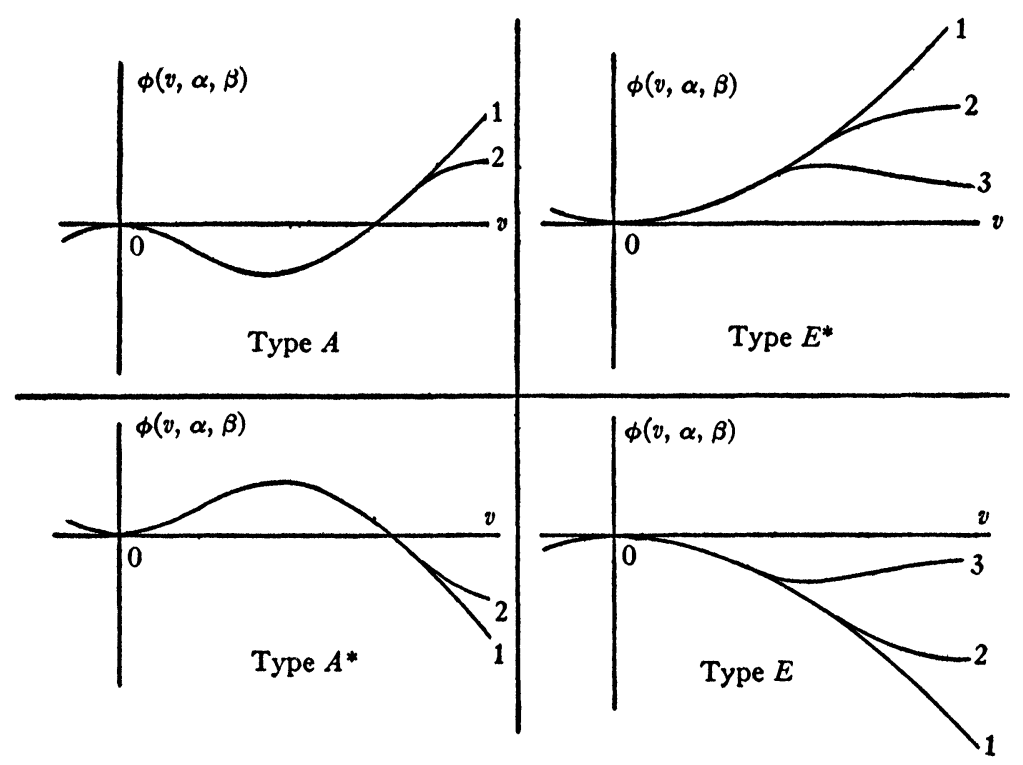

FIG. 2

In figure 2 we have sketched ten curves $\phi=\phi(v, \alpha, \beta)$ in the $v \phi$-plane which exhibit these properties. We shall call these curves the characteristic curves for the sets and subsets of figure 1. The lettering in the two figures is such that the curve marked 1 in type $A$ exhibits the invariant properties of $\phi(v, \alpha, \beta)$ for $(\alpha, \beta)$ in the set marked $A_{1}$, and so on. A brief description of the curves and an exact definition of the point set for each curve follows.

Type $A$. The characteristic curves for set $A$ (where $\psi_{2}<\beta<\psi_{3}$ ) have the common properties: a maximum for $v=0$, a minimum and a zero for positive values of $v$, and a positive limit as $v \rightarrow \infty$. $A$ has two subsets:

and

$$
A_{1}:-\infty<\alpha<-2, \psi_{2}<\beta<\psi_{3} \text {, }
$$




$$
A_{2}:-1 / 2<\alpha<+1, \psi_{2}<\beta<\psi_{3} .
$$

In $A_{1}, \lim _{v \rightarrow \infty} \phi(v, \alpha, \beta)=\infty$, but in $A_{2}$ the limit is finite.

Type $A^{*}$. The characteristic curves for set $A^{*}$ (where $\psi_{3}<\beta<\psi_{2}$ ) have properties as would a reflection of type $A$ in the $v$-axis. $A^{*}$ has two subsets:

and

$$
A_{1}^{*}:-2<\alpha \leqq-1, \psi_{3}<\beta<\psi_{2},
$$

$$
A_{2}{ }^{*}:-1<\alpha<-1 / 2,1<\alpha<\infty, \psi_{3}<\beta<\psi_{2} .
$$

Type $E E^{*}$. The characteristic curve for the set $E E^{*}$ is the horizontal axis or $v$-axis, since for $(\alpha, \beta)$ in $E E^{*}:(-2,0),(-1 / 2,1 / 2),(1,1)$, $\phi(v, \alpha, \beta) \equiv 0$.

Type E. The characteristic curves for the set $E-E E^{*}$ have the common properties: a maximum at the origin, and negative values for all $v$ different from zero. There are three subsets:

$E_{1}: \alpha \leqq-1, \beta \geqq \max \left(\psi_{2}, \psi_{3}\right)$ except for the point $(-2,0)$ in $E E^{*}$,

$E_{2}:-1<\alpha, \beta \geqq \max \left(\psi_{2}, \psi_{4}\right)$ except for the points $(-1 / 2,1 / 2)$ and $(1,1)$ in $E E^{*}$.

$E_{3}:-1 / 2<\alpha<1, \psi_{3} \leqq \beta<\psi_{4}$.

In $E_{1}, \lim _{v \rightarrow \infty} \phi(v, \alpha, \beta)=-\infty$. In $E_{2}$ and $E_{3}$ the limit is finite, and $E_{3}$ is distinguished by being the only subset of $E-E E^{*}$ in which the function has a minimum. When $\beta=\psi_{3}$ in $E_{3}, \lim _{v \rightarrow \infty} \phi(v, \alpha, \beta)$ is zero.

Type $E^{*}$. The characteristic curves for $E^{*}-E E^{*}$ have common properties as would a reflection of type $E$ in the $v$-axis. $E^{*}-E E^{*}$ has three subsets:

$E_{1}^{*}:-\infty<\alpha<+\infty, \beta \leqq \min \left(\psi_{2}, 0\right)$ except for the point $(-2,0)$,

$E_{2}{ }^{*}:-1<\alpha<+\infty, 0<\beta \leqq \min \left(\psi_{2}, \psi_{4}\right)$ except for $(-1 / 2,1 / 2)$ and $(1,1)$.

$E_{3}{ }^{*}:-1<\alpha<-1 / 2, \psi_{4}<\beta \leqq \psi_{3} ;$ and $1<\alpha<+\infty, \psi_{4}<\beta \leqq \psi_{3}$.

10. The sets $E_{M}$ and $E_{M}^{*}$. Radó showed [1, p. 274] that when:

(1) $f(x)$ is a general positive and continuous convex function,

(2) $l(x)$ is the linear function that coincides with $f(x)$ at $x-h$ and $x+h$,

(3) $l(x)$ satisfies the inequality (1.1), then $f(x)$ satisfies (1.1).

Now for any given $M>0$, the class $K K_{M}$ is a restricted class of convex functions $f(x)$, containing only those for which $2^{-1} \log (B / b) \leqq M$.

Let us now consider the equation, for any given $M>0$,

$$
\phi(M, \alpha, \beta)=0,
$$

where $\phi$ is the function defined in (3.3). We may think of $\beta$ in(10.1) 
as an implicit function of $\alpha$, with $M$ as a parameter, which we shall denote by $\beta(\alpha, M)$. The curve $\beta=\beta(\alpha, M)$ in the $\alpha \beta$-plane lies entirely in the set where $(\alpha, \beta)$ satisfies the inequalities (8.2) and passes through the points of $E E^{*}$ for all $M$. For small $M$, the curve lies close to the straight line $\beta=\psi_{2}(\alpha)$; in fact, using (5.1), replacing $v$ by $M$, setting $\phi(M, \alpha, \beta)=0$, dividing by $M^{2}$, we find that $\beta \rightarrow(\alpha+2) / 3$ as $M \rightarrow 0$. For large $M$, the curve lies close to $\beta=\psi_{3}(\alpha)$. Approximate values of $\beta$ for given values of $\alpha$ and $M$ may be easily computed by the use of tables.

We conclude with the theorems:

Theorem 10.2. A pair $(\alpha, \beta)$ belongs to $E_{M}$ if and only if $\beta \geqq \max \left(\psi_{2}, \beta(\alpha, M)\right)$.

TheOREM 10.3. A pair $(\alpha, \beta)$ belongs to $E_{M^{*}}$ if and only if $\beta \leqq \min \left(\psi_{2}, \beta(\alpha, M)\right)$.

We first prove the sufficiency of the condition in Theorem 10.2. Our hypotheses are: (1) $f(x)$ is in $K,(2) f(x)$ is in $K_{M}$, and (3) the pair $(\alpha, \beta)$ satisfies $\beta \geqq \max \left(\psi_{2}, \beta(\alpha, M)\right)$. We wish to prove that: (4) $f(x)$ is in $K_{\alpha \beta}$.

By (3) the pair $(\alpha, \beta)$ is either in the set $E$ where $\beta \geqq \max \left(\psi_{2}, \psi_{3}\right)$ or in that part of the set $A$ where $\beta \geqq \beta(\alpha, M)$. If $(\alpha, \beta)$ is in $E$ it is obviously in $E_{M}$ and $f(x)$ is in $K_{\alpha \beta}$. If $(\alpha, \beta)$ is in that part of $A$ where $\beta \geqq \beta(\alpha, M)$ we consider first $\beta=\beta(\alpha, M)$ and then $\beta>\beta(\alpha, M)$.

When $\beta=\beta(\alpha, M), \phi(M, \alpha, \beta)=0$. For the $l(x)$ that coincides with $f(x)$ at $x-h$ and $x+h, A(l, x, h, \beta)=A(f, x, h, \beta)$, and by (1), $I(f, x, h, \alpha) \leqq I(l, x, h, \alpha)$. For the same $l(x), v \leqq 2^{-1} \log (B / b) \leqq M$, and therefore $\phi(v, \alpha, \beta) \leqq 0$, for $f(x)$ is in $K_{M}$, and the pair $(\alpha, \beta)$ that we are considering is in $A$. Hence we have $I(l, x, h, \alpha) \leqq A(l, x, h, \beta)$ and thus $I(f, x, h, \alpha) \leqq A(f, x, h, \beta)$, which proves that $f(x)$ is in $K_{\alpha \beta}$. When $\beta>\beta(\alpha, M), \phi(M, \alpha, \beta)<0$, for $A(f, x, h, \beta)$ is an increasing function of $\beta[4$, p. 26], and therefore $\phi(M, \alpha, \beta)$ deccreases with increase in $\beta$. Thus for all $v \leqq M, \phi(v, \alpha, \beta) \leqq 0$, and $f(x)$ is in $K_{\alpha \beta}$.

To prove the necessity of the condition we have as hypotheses: (1), (2) and (4), and wish to prove (3). Suppose, contrary to our conclusion, for all $f(x)$ in $K K_{M}$, there exists a $\beta<\max \left(\psi_{2}, \beta(\alpha, M)\right)$ such that $f(x)$ is in $K_{\alpha \beta}$. In $A$, where $\beta(\alpha, M)>\psi_{2}(\alpha), \beta<\beta(\alpha, M)$ implies that $\phi(M, \alpha, \beta)>0$. In $A^{*}$, where $\beta(\alpha, M)<\psi_{2}(\alpha), \beta<\psi_{2}(\alpha)$ implies that for sufficiently small $v, \phi(v, \alpha, \beta)>0$. In $E^{*}-E E^{*}$, for all $v$, $\phi(v, \alpha, \beta)>0$. Thus in any case, we have a value of $v \leqq M$ for which $\phi(v, \alpha, \beta)>0$. Hence for some $v \leqq M$, the inequality (1.1) is not satisfied, in particular, by a linear function in the class $K K_{M}$, which gives us a contradiction. 
The proof of Theorem 10.3 is similar, but with obvious modifications.

\section{REFERENCES}

1. Tibor Rad6, On convex functions, Trans. Amer. Math. Soc. vol. 37 (1935) pp. $266-285$.

2. H. B. Dwight, Tables of integrals and other mathematical data, Macmillan, 1934.

3. G. P6lya and G. Szegö, Aufgaben und Lehrsätze aus der Analysis, vol. 2, Berlin, Springer, 1925.

4. G. H. Hardy, J. E. Littlewood, and G. Pólya, Inequalities, Cambridge, 1934.

Emmanuel Missionary College

\section{A SIMPLE SUFFICIENT CONDITION THAT A METHOD OF SUMMABILITY BE STRONGER THAN CONVERGENCE}

\section{RALPH PALMER AGNEW}

1. Introduction. A matrix $a_{n k}$ of real or complex constants determines a transformation

$$
\sigma_{n}=\sum_{k=1}^{\infty} a_{n k} s_{k}
$$

and a method $A$ of summability by means of which a given sequence $s_{1}, s_{2}, \ldots$ is summable to $\sigma$ if the series in (1) converge and define numbers $\sigma_{1}, \sigma_{2}, \cdots$ such that $\sigma_{n} \rightarrow \sigma$ as $n \rightarrow \infty$. If a sequence $s_{n}$ is summable $A$, we say that $A\left\{s_{n}\right\}$ exists and that $s_{n}$ belongs to the summability field of $A$. If $s_{n}$ is summable $A$ to $\sigma$, we say that $A\left\{s_{n}\right\}=\sigma$. The method $A$ is regular if $A\left\{s_{n}\right\}=\lim s_{n}$ whenever $\lim s_{n}$ exists.

Toeplitz [1911] (reference in bibliography at end of this paper) proved that $A$ is regular if and only if the three conditions

$$
\begin{aligned}
\sum_{k=1}^{\infty}\left|a_{n k}\right| & \leqq M, & n=1,2,3, \cdots, \\
\lim _{n \rightarrow \infty} a_{n k} & =0, & k=1,2,3, \cdots, \\
\lim _{n \rightarrow \infty} \sum_{k=1}^{\infty} a_{n k} & =1 &
\end{aligned}
$$

Presented to the Society, November 24, 1945; received by the editors September 8 , 1945. 